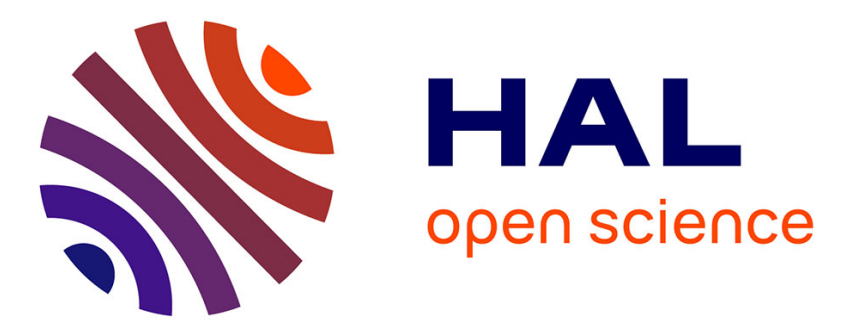

\title{
Effects of anisotropy and size of polar nano thin films on their thermal conductivity due to surface phonon-polaritons
}

Jose Ordonez-Miranda, Laurent Tranchant, Beomjoon Kim, Yann Chalopin, Thomas Antoni, Sebastian Volz

\section{To cite this version:}

Jose Ordonez-Miranda, Laurent Tranchant, Beomjoon Kim, Yann Chalopin, Thomas Antoni, et al.. Effects of anisotropy and size of polar nano thin films on their thermal conductivity due to surface phonon-polaritons. Japanese Journal of Applied Physics, part 2: Letters, 2014, pp.24. 10.7567/APEX.7.035201 . hal-01134043

HAL Id: hal-01134043

https://hal-centralesupelec.archives-ouvertes.fr/hal-01134043

Submitted on 24 Mar 2015

HAL is a multi-disciplinary open access archive for the deposit and dissemination of scientific research documents, whether they are published or not. The documents may come from teaching and research institutions in France or abroad, or from public or private research centers.
L'archive ouverte pluridisciplinaire HAL, est destinée au dépôt et à la diffusion de documents scientifiques de niveau recherche, publiés ou non, émanant des établissements d'enseignement et de recherche français ou étrangers, des laboratoires publics ou privés. 


\title{
Anisotropy and size effects on the thermal conductivity of polar nano thin films due to surface phonon-polaritons
}

\author{
Jose Ordonez-Miranda, ${ }^{1}$ Laurent Tranchant, ${ }^{1}$ Beomjoon Kim, ${ }^{2}$ \\ Yann Chalopin, ${ }^{1}$ Thomas Antoni, ${ }^{1,3}$ and Sebastian Volz ${ }^{1, *}$ \\ ${ }^{1}$ Laboratoire d'Énergétique Moléculaire et Macroscopique, \\ Combustion, UPR CNRS 288, École Centrale Paris, \\ Grande Voie des Vignes, 92295 Châtenay-Malabry, France. \\ ${ }^{2}$ CIRMM, Institute of Industrial Science, University of Tokyo, Japan. \\ ${ }^{3}$ École Centrale Paris, Laboratoire de Photonique Quantique et Moléculaire, \\ CNRS (UMR 8537), École Normale Supérieure de Cachan, \\ Grande Voie des Vignes, F-92295 Châtenay-Malabry cedex, France.
}

(Dated: August 15, 2013)

\begin{abstract}
The effects of the permittivity anisotropy and size of a nano thin film on its thermal conductivity due to surface phonon-polaritons are studied. We demonstrate that this thermal conductivity is a linear combination of the inverse first and third powers of the film thickness. For a 100 nm-thick film of $\alpha$-quartz surrounded by air, it is shown that its thermal conductivity along the optical axis is comparable to the phonon counterpart and equals to $13 \mathrm{~W} / \mathrm{m} . \mathrm{K}$, which is $25 \%$ higher than that along a perpendicular direction, at room temperature. Higher values are found for thinner films at higher temperatures.
\end{abstract}

Surface phonon-polaritons (SPPs) are electromagnetic waves generated by the coupling between photons and phonons at the interface between two media [1-3]. Over the past few years, various research groups have shown that these surface waves have promising applications on the thermal performance of nanoscale devices [4-6], radiative heat transfer[7-10], high-density infrared data storage [11], surface infrared absorption,[12] coherent thermal emission, [13] and photonics [14, 15]. In these cases, the operating principles are based on the fact that in nanomaterials, the surface effects predominate over the volumetric ones, and therefore the energy transport by SPPs is particularly important. The SPP energy contribution increases as the material size is scaled-down in the direction perpendicular to the propagation one $[4,6]$.

The nature of SPPs propagating along the interface of isotropic media is now well understood. SPPs can have long propagation lengths, of the order of a few centimeters on thin films [4-6], which in turn could lead to an efficient energy transport. Based on the Boltzmann transport equation, Chen et al.[4] showed that the SPP thermal conductivity of a $40 \mathrm{~nm}$-thick thin film of silicon dioxide suspended in air, is $4 \mathrm{~W} / \mathrm{m} . \mathrm{K}$ at $500 \mathrm{~K}$. This value represents an increase of about $100 \%$ over its bulk phonon thermal conductivity. This result has been confirmed by the fluctuation-dissipation theorem [16] and is consistent with experimental data for the propagation length [5]. More recently and under a fully analytical approach, we reconfirmed previous numerical results [4], for the SPP propagation length and thermal conductivity [6], and showed that a substrate with a permittivity slightly different than that of the superstrate of a nano thin film can generate large propagation lengths and therefore en-

\footnotetext{
* Corresponding author: sebastian.volz@ecp.fr
}

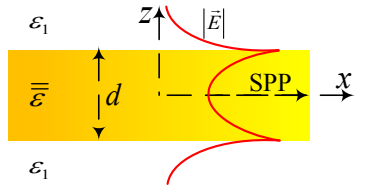

FIG. 1. Cross-section of the thin film under consideration.

hance significantly its SPP thermal conductivity. Given that the absorption of energy by the film and its surrounding media is determined by their permittivities, it is expected that thin films with anisotropic permittivity can provide more variables than isotropic ones to control and optimize the SPP energy transport.

The purpose of this letter is to analyze the effects of the anisotropic permittivity and size of biaxial crystalline thin films on their SPP thermal conductivity. For nano thin films, an explicit expression for the SPP thermal conductivity as a function of their thickness is obtained, which provides clear physical insights on how the heat transport depends on the permittivity of the thin film and its surrounding media. The SPP propagation along the principal axes of the crystal are discussed in detail.

Let us consider a layered system supporting the propagation of SPPs, as shown in Fig. 1. The thin film is a biaxial crystal described by its permittivity tensor

$$
\overline{\bar{\varepsilon}}=\left(\begin{array}{ccc}
\varepsilon_{x} & 0 & 0 \\
0 & \varepsilon_{y} & 0 \\
0 & 0 & \varepsilon_{z}
\end{array}\right),
$$

where $\varepsilon_{j}$ for $j=x, y, z$ are the frequency-dependent permittivities along the principal axes of the anisotropic crystal. The surrounding medium on either side has a constant permittivity $\varepsilon_{1}>0$, as is the case of air, within a wide range of frequency [17].

By solving the Maxwell equations under proper bound- 
ary conditions for the transverse magnetic polarization required for the existence of surface polaritons [4], the following dispersion relation for the wave vector $\beta$, along the $x$ axis is obtained

$$
\tanh \left(p_{2} d / 2\right)=-\frac{\varepsilon_{1} p_{2}}{\varepsilon_{x} p_{1}},
$$

where the transverse wave vectors inside and outside the film are given by $p_{2}^{2}=\left(\varepsilon_{x} / \varepsilon_{z}\right)\left(\beta^{2}-\varepsilon_{z} k_{0}^{2}\right)$ and $p_{1}^{2}=\beta^{2}-\varepsilon_{1} k_{0}^{2}$, respectively; $k_{0}=\omega / c, \omega$ is the excitation frequency and $c$ is the speed of light in vacuum. Note that the dispersion relation in Eq. (2) depends on the permittivity along $\left(\varepsilon_{x}\right)$ and across $\left(\varepsilon_{z}\right)$ the direction of SPP propagation, but not on the permittivity along the $y$ axis, because the SPPs do not propagate in this direction. Equation (2) can significantly be simplified for a thin film $\left(\left|p_{2}\right| d<<2\right)$, which is of interest in this work to enhance the SPP propagation along the film. For a nano thin film of $\alpha$-quartz, this condition is well satisfied for $d \leq 300 \mathrm{~nm}$. In this case, Eq. (2) reduces to

$$
\frac{p_{2}^{2}}{\varepsilon_{x}}+\frac{2}{d} \frac{p_{1}}{\varepsilon_{1}}=0
$$

which, given the definition of $p_{2}$, depends only on the permittivity $\varepsilon_{z}$ along the axis perpendicular to the film plane. As the film thickness is scaled down, the coupling of the electric and magnetic fields traveling along the two interfaces of the film becomes stronger, and therefore the dispersion relations of these fields should become more sensitive to $\varepsilon_{z}$ than to $\varepsilon_{x}$. This shows that the separation of the permittivities in the dispersion relation of nano thin films is reasonable. Equation (3) is equal to our corresponding previous result for isotropic media after the substitution $\varepsilon_{z} \rightarrow \varepsilon_{2}$ [6]. Therefore, based on the analytical expressions for the real part $\beta_{R}$ of $\beta=\beta_{R}+i \beta_{I}$ and propagation length $\Lambda=1 /\left(2 \beta_{I}\right)$ derived for this case [6], the SPP thermal conductivity can be written as

$$
\kappa=\frac{A}{d^{3}}+\frac{B}{d},
$$

where

$$
\begin{gathered}
A=\frac{1}{2 \pi} \int_{0}^{\infty} \frac{\hbar \omega}{\xi k_{0}^{2}} \frac{\left|\varepsilon_{z}\right|^{2}}{\varepsilon_{1}^{2} \varepsilon_{I}} \frac{\partial f}{\partial T} d \omega \\
B=\frac{1}{8 \pi} \int_{0}^{\infty} \hbar \omega\left(\frac{\xi}{\varepsilon_{1} \varepsilon_{I}}-\frac{\varepsilon_{1} \varepsilon_{I}}{\xi}\right) \frac{\partial f}{\partial T} d \omega
\end{gathered}
$$

being $\varepsilon_{z}=\varepsilon_{R}+i \varepsilon_{I}$ and $\xi=\left|\varepsilon_{z}\right|^{2}-\varepsilon_{1} \varepsilon_{R}$, which must be positive for any $\omega>0$, to guarantee the propagation of SPPs $(\Lambda>0)$. Within the thin film limit $\left(\left|p_{2}\right| d<<2\right)$, the simple Eq.(4) establishes that the increase of the SPP thermal conductivity as the film thickness decreases is totally determined by the material-dependent parameters $A$ and $B$. When $\sqrt[3]{A}<<d<B, \kappa$ is driven by the inverse of the film thickness; while for $B<<d<\sqrt[3]{A}, \kappa$ is inversely proportional to the cube of this thickness. The smaller values of the film thickness $d$ are set by the surface
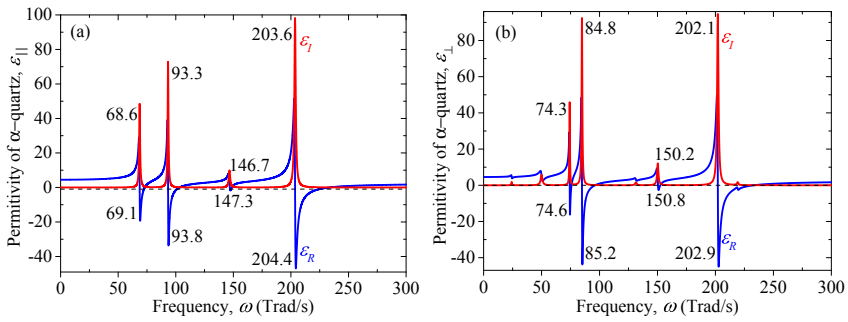

FIG. 2. Real $\left(\varepsilon_{R}\right)$ and imaginary $\left(\varepsilon_{I}\right)$ parts of the permittivity (a) parallel and (b) perpendicular to the optical axis of $\alpha$ quartz as a function of frequency.

roughness height and peak-to-peak distance, which are typically of the order of $1 \mathrm{~nm}$. [4] Note that both parameters $A$ and $B$, and hence the SPP thermal conductivity, increase as the permittivity $\varepsilon_{1}$ of the surrounding media decreases [4]. This result agrees with previous numerical simulations and is due to the fact that the propagation length $\Lambda$ increases with $\varepsilon_{1}[6]$.

The quantification of the SPP thermal conductivity and its dependence on the anisotropic permittivity and size are now analyzed for a thin film of $\alpha$-quartz surrounded by air $\left(\varepsilon_{1}=1\right)$. This material is crystalline silica, which is common in nature and applications $[1,18]$. The permittivities in the direction parallel $(j=\|)$ and perpendicular $(j=\perp)$ to its optical axis are given by [18]

$$
\varepsilon_{j}(\omega)=\varepsilon_{j}^{\infty} \prod_{n=1}^{N_{j}}\left(\frac{\Omega_{j n}^{2}-\omega^{2}-i \gamma_{j n} \omega}{\omega_{j n}^{2}-\omega^{2}-i \gamma_{j n} \omega}\right),
$$

where $\Omega_{j n}$ and $\omega_{j n}$ are the longitudinal and transverse optical frequencies, respectively; $\gamma_{j n}$ is a damping constant and $\varepsilon_{j}^{\infty}$ is the high frequency permittivity. The experimental values of these parameters for the $N_{\|}=4$ $\left(N_{\perp}=8\right)$ vibrational branches parallel (perpendicular) to the optical axis of $\alpha$-quartz are reported in the literature $[18,19]$. Based on Eq. (6), it can be shown that the maxima of the imaginary part of $\varepsilon_{j}$ occurs at $\omega=\omega_{j n}$, for $\omega_{j n}>>\gamma_{j n}$, as is the case of $\alpha$-quartz and most crystals. This indicates that these materials absorb significant energy from the electromagnetic field at those frequencies.

Figures 2(a) and 2(b) show the frequency dependence of the real and imaginary parts of the permittivity parallel $\left(\varepsilon_{\|}\right)$and perpendicular $\left(\varepsilon_{\perp}\right)$ to the optical axis of $\alpha$-quartz, respectively. The sharp resonance peaks of the imaginary parts of both $\varepsilon_{\|}$and $\varepsilon_{\perp}$, along with their small values for broad frequency ranges, show that the SPPs can propagate over relatively large distances within these frequency intervals. $\varepsilon_{\|}$has a less number of absorbing peaks than $\varepsilon_{\perp}$, and hence the SPP energy transport is expected to be more efficient along the optical axis $\left(\varepsilon_{x}=\varepsilon_{\|}\right)$than along the perpendicular axis $\left(\varepsilon_{x}=\varepsilon_{\perp}\right)$.

The dispersion relation and propagation length of SPPs traveling along the film/air interface are shown in Figs. 3(a) and 3(b), respectively. The displayed range of frequencies is determined by the conditions of existence $\left(\operatorname{Re}\left(p_{l}\right)>0\right.$, for $\left.l=1,2\right)$ and propagation $(\Lambda>0)$ 

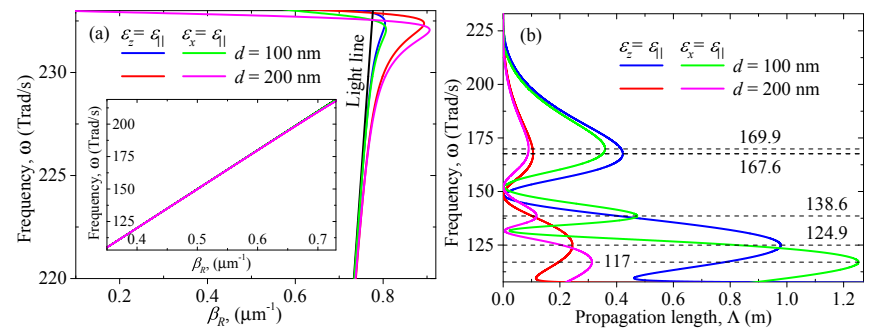

FIG. 3. (a) Dispersion relation and (b) propagation length as a function of the frequency, for a thin film of $\alpha$-quartz surrounded by air.

of SPPs. For SPPs traveling along the optical axis of $\alpha$-quartz $\left(\varepsilon_{x}=\varepsilon_{\|}\right.$and $\left.\varepsilon_{y}=\varepsilon_{z}=\varepsilon_{\perp}\right)$, this range is $101 \mathrm{Trad} / \mathrm{s}<\omega<233 \mathrm{Trad} / \mathrm{s}$; while for SPP propagating along the axis of $\varepsilon_{\perp}\left(\varepsilon_{x}=\varepsilon_{\perp}\right.$ and $\left.\varepsilon_{y}=\varepsilon_{z}=\varepsilon_{\|}\right)$, the frequency interval is $108 \mathrm{Trad} / \mathrm{s}<\omega<233 \mathrm{Trad} / \mathrm{s}$. Note that for both propagation directions, $\beta_{R}$ decreases and approaches the light line, as the frequency decreases. This indicates a photon-like nature of the SPPs. As the frequency increases, the dispersion relation separates from the light line and tends to a phonon-like behavior. This feature strengths when the film thickness increases. The corresponding propagation lengths in Fig. 3(b) take larger values at the frequencies where the absorption of energy is minimal (Fig.2). For each propagation direction, the maxima of $\Lambda$ are independent of $d$ and depends on both the real and imaginary parts of the respective permittivity, as was shown previously [6]. This is why the minimum of the propagation lengths are not placed exactly at the resonance frequency of $\varepsilon_{I}$, but rather in its vicinity. Note also that for a given film thickness, larger propagation lengths are obtained when the optical axis is parallel to the SPP direction.

The real parts of the transverse wave vectors inside and outside of the thin film are positive, as shown in Fig. 4(a). This fact along with Figs. 3(b) reveals that the propagation of SPPs is present in a broad band of frequencies. This is further confirmed by the distribution of the Poynting vector shown in Fig. 4(b). The energy flux propagating inside the film is close to zero and negligible in comparison with that outside of it, due to the very small absorption by the surrounding air. The high concentration of energy at the interface enables the absorbing film to support the propagation of SPPs. As a result of the coupling of the electromagnetic fields traveling along both film interfaces, the energy flux increases as the film thickness decreases. This indicates that the the SPP thermal conductivity should increase as the film thickness reduces, which is consistent with Eq. (4) and Fig. 3(b). For the frequency $\omega=169.9 \mathrm{Trad} / \mathrm{s}$ and thickness $d=200 \mathrm{~nm}$, Figs. 3(b) and 4(b) show that the SPPs propagate about $100 \mathrm{~mm}$ along the thin film and just $100 \mu \mathrm{m}$ across it. This difference on three orders of magnitude shows the promising potential of SPPs to transport energy along thin films.

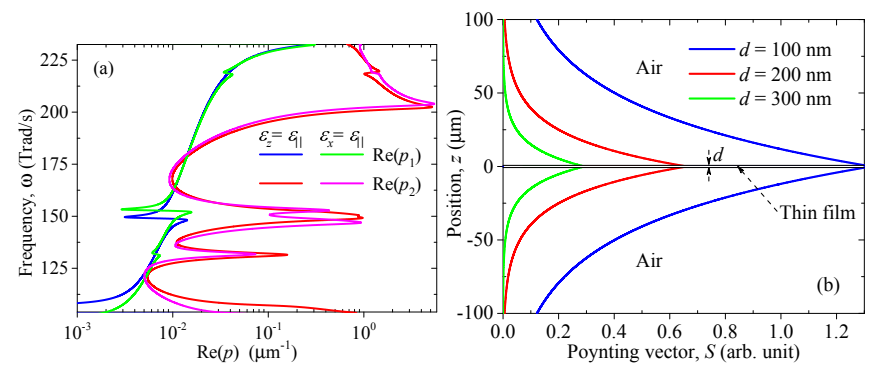

FIG. 4. Frequency dependence of $\operatorname{Re}\left(p_{j}\right)$ inside and outside of a $100 \mathrm{~nm}$-thick film of $\alpha$-quartz surrounded by air. (b) Poynting vector distribution at $x=0$ and $\omega=169.9 \mathrm{Trad} / \mathrm{s}$, for SPPs propagating along the optical axis.

The integrand of the thermal conductivity defined in Eq. 4 as a function of the frequency is shown in Fig. 5(a), for SPP propagation along two principal axes of the $\alpha$ quartz film. Note that the oscillatory behavior of these spectra is determined by the permittivity, and their maxima coincides with the corresponding ones of the propagation lengths shown in Fig. 3(b). The major contributions to the SPP thermal conductivity (area under the curve) arises from the vicinity of these peaks, as shown by the cumulative thermal conductivity in Fig. 5(b). Note that the increase of the thermal conductivity exhibits an inflection point at the frequency where the maxima of $\Lambda$ occur. The SPP thermal conductivity $\kappa$ increases as the film thickness decreases, and it can be much larger than its phonon counterpart for a film thickness smaller than $170 \mathrm{~nm}$, as shown in Fig. 5(c). It is clear that $\kappa$ becomes significant for nano thin films and negligible for micro thin films, as reported previously $[4,6] . \kappa$ is higher when the SPPs propagate along the principal axis with smaller absorption (the optical axis, in this case), as expected. The enhancement of $\kappa$, due to the permittivity anisotropy of $\alpha$-quartz, increases for smaller film thicknesses and lower temperatures. For $d=100 \mathrm{~nm}$, it passes from $25 \%$ at $T=300 \mathrm{~K}$ to $14 \%$ at $T=500 \mathrm{~K}$. Thus, it is clear that $\kappa$ is fairly sensitive to the propagation direction and temperature of the anisotropic film. In contrast to the phonon thermal conductivity of $\alpha$-quartz, the SPP one increases with temperature (Fig. 5(d)). This shows that the SPPs have the potential to diminish the overheating of materials under high temperatures of operation, by increasing their thermal conductivity.

The source of the high SPP thermal conductivity is the propagation length at small wavevectors, which are very close to the light line, as shown in Figs. 3(a) and 3 (b). The largest propagation length in this photon-like regime is about $1 \mathrm{~m}$ for a $100 \mathrm{~nm}$-thick film of $\alpha$-quartz. This long value of $\Lambda$ implies that only thin films with a length $(L)$ longer than this distance $(L>>\Lambda)$ can benefit from the enhanced SPP thermal conductivity. In practice, however, $L$ is not so long and therefore the propagation length has to be redefined. For this case, the Matthiessen's rule [20], establishes that the effective propagation length $\left(\Lambda_{e}\right)$ is given by $\Lambda_{e}^{-1}=\Lambda^{-1}+L^{-1}$. 

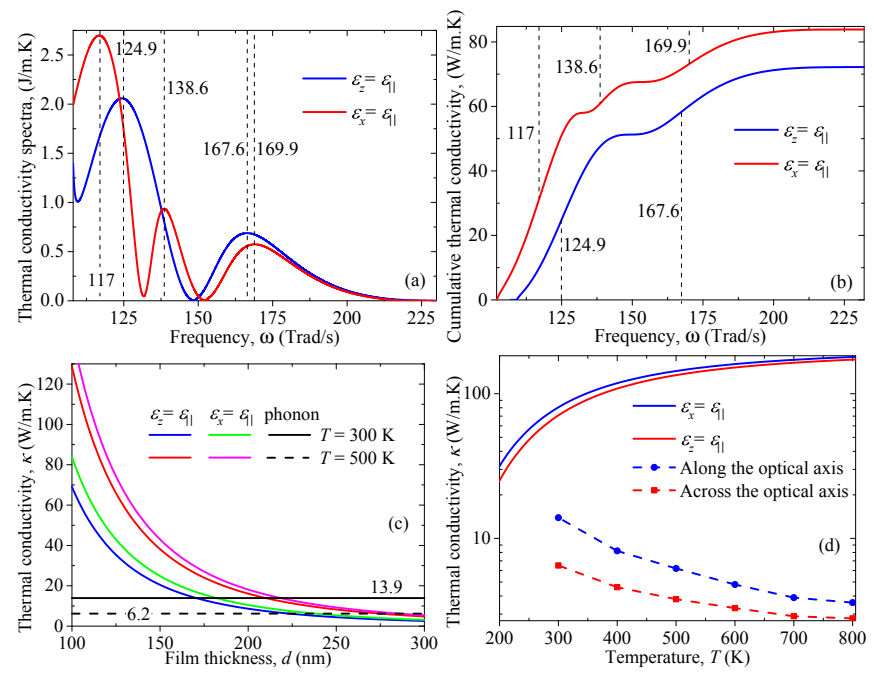

FIG. 5. (a) Thermal conductivity spectrum and (b) its cumulative values for $d=100 \mathrm{~nm}$ and $T=300 \mathrm{~K}$. Thermal conductivity as a function of the film (c) thickness and (d) temperature $(d=100 \mathrm{~nm})$. The experimental points stand for the phonon counterpart of $\alpha$-quartz along its optical axis.

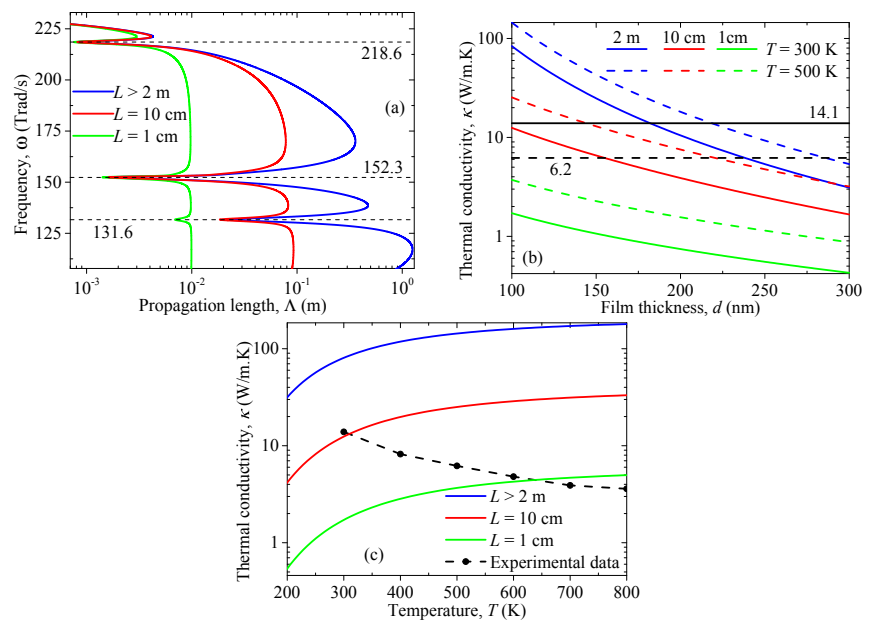

FIG. 6. (a) Frequency dependence of the propagation length for $T=300 \mathrm{~K}$ and thermal conductivity as a function of the (b) film thickness and (c) temperature, for three film lengths. The film thickness in (a) and (c) is $d=100 \mathrm{~nm}$.
Thus, the effect of the film length $L$ on the SPP energy transport appears through the reduction of the intrinsic propagation length $\Lambda$. To quantify the correspondin reduction of the SPP thermal conductivity, we use the explicit expression for $\kappa$ in terms of the propagation length $[4,6]$.

Figure 6(a) shows the effective propagation length of SPPs propagating along the optical axis of the thin film, for three lengths $L$. The case $L>2 \mathrm{~m}$ corresponds to $\Lambda_{e}=\Lambda$. Note that $\Lambda_{e}$ follows the same trend than $\Lambda$ and its maximum values are smaller but comparable to $L$. This reduction of the propagation length from meters to centimeters has a strong impact on the SPP thermal conductivity $\kappa$, as shown in Fig. $6(\mathrm{~b})$. For $T=300$ $\mathrm{K}, \kappa$ reduces from $82 \mathrm{~W} / \mathrm{m} . \mathrm{K}$ to $13 \mathrm{~W} / \mathrm{m} . \mathrm{K}$ when the film length is scaled down from $L>2 \mathrm{~m}$ to $L=10$ $\mathrm{cm}$. Despite of this sizeable reduction of $\kappa$, its value is only $1.1 \mathrm{~W} / \mathrm{m} . \mathrm{K}$ smaller than its phonon counterpart, which shows that the SPPs are still able to significantly enhance the phonon thermal conductivity of nano thin films. Even though lower values of $\kappa$ are obtained for a smaller film length $(L=1 \mathrm{~cm})$, this reduction can be offset at higher temperature, as shown in Fig. 6(c). In summary, the energy transport of SPPs along nano thin films can be optimized by choosing the propagation direction along the axis of less absorption, decreasing the film thickness, increasing the film length and raising the temperature. A simple expression for the SPP thermal conductivity as a linear combination of the inverse first and third powers of the film thickness has been derived. The thermal conductivity along the optical axis of a 100 nm-thick film of $\alpha$-quartz is $25 \%$ higher than that along a perpendicular direction. A thermal conductivity of 13 $\mathrm{W} / \mathrm{m} . \mathrm{K}$ has been obtained for a thin film of $100 \mathrm{~nm}$ in thickness and $10 \mathrm{~cm}$ in length, at room temperature.
[1] H. J. Bakker, S. Hunsche, and H. Kurz, Rev. Mod. Phys. 70, 523 (1998).

[2] N. Dahan, A. Niv, G. Biener, V. Kleiner, and E. Hasman, Opt. Lett. 33, 3195 (2005).

[3] B. Neuner et al., Opt. Lett. 34, 2667 (2009).

[4] D. Z. A. Chen, A. Narayanaswamy, and G. Chen, Phys. Rev. B 72, 155435 (2005).

[5] D. Z. A. Chen and G. Chen, Appl. Phys. Lett. 91, 121906 (2007).

[6] J. Ordonez-Miranda et al., J. Appl. Phys. 113, 084311 (2013).

[7] M. Francoeur, M. P. Menguc, and R. Vaillon, Appl.
Phys. Lett. 93, 043109 (2008).

[8] P. O. Chapuis, M. Laroche, S. Volz, and J. J. Greffet, Appl. Phys. Lett. 92, 201906 (2008).

[9] G. Domingues, S. Volz, K. Joulain, and J. J. Greffet, Phys. Rev. Lett. 94, 085901 (2005).

[10] P. O. Chapuis, S. Volz, C. Henkel, K. Joulain, and J. J. Greffet, Phys. Rev. B 77, 035431 (2008).

[11] N. Ocelic and R. Hillenbrand, Nature Mater. 3, 606 (2004).

[12] H. C. Kim and X. Cheng, Opt. Lett. 35, 3748 (2010).

[13] J. J. Greffet et al., Nature 416, 61 (2002).

[14] T. Taubner, F. Keilmann, and R. Hillenbrand, Nano 
Lett. 4, 1669 (2004).

[15] E. Rousseau et al., Nature Photonics 3, 514 (2009).

[16] D. Z. A. Chen and G. Chen, Front. Heat Mass Transfer 1, 023005 (2010).

[17] E. D. Palik, Handbook of Optical Constants of Solids (Academic Press, Orlando, Florida, 1985).
[18] G. Lamprecht and L. Merten, Phys. Stat. Sol. (b) 55, 33 (1973).

[19] L. Merten and G. Lamprecht, Phys. Stat. Sol. (b) 39, 573 (1970).

[20] J. M. Ziman, Electrons and phonons: the theory of transport phenomena in solids (Clarendon Press, Oxford, 2001). 